\title{
Activity Tracking and Indoor Positioning with a Wearable Magnet
}

\section{Andrei Popleteev}

University of Luxembourg

4, rue Alphonse Weicker

L-2721 Luxembourg

andrei.popleteev@uni.lu
Permission to make digital or hard copies of part or all of this work for personal or classroom use is granted without fee provided that copies are not made or distributed for profit or commercial advantage and that copies bear this notice and the full citation on the first page. Copyrights for third-party components of this work must be honored. For all other uses, contact the Owner/Author.

Copyright is held by the owner/author(s).

Ubicomp/ISWC'15 Adjunct, September 07-11, 2015, Osaka, Japan ACM 978-1-4503-3575-1/15/09.

http://dx.doi.org/10.1145/2800835.2800938

\author{
Abstract \\ This paper presents an unconventional application of \\ digital compass sensors for localization and activity \\ monitoring in ambient assisted living scenarios. \\ Benefits and limitations of the proposed approach are \\ reviewed and compared to these of traditional tracking \\ methods, such as wearable devices, surveillance \\ cameras and device-free localization.
}

\section{Author Keywords}

Digital compass; magnetometer; activity recognition; indoor localization.

\section{ACM Classification Keywords}

H.5.m. Information interfaces and presentation:

Miscellaneous

\section{Introduction}

Monitoring physical activity and user location is an important component of smart context-aware environments and ubiquitous computing in general. To enable such monitoring, a number of sensing

techniques have been proposed, such as smartphonebased tracking, wearable sensors, and video analysis from surveillance cameras. However, these methods have their limitations, such as poor indoor localization accuracy, battery life, computational requirements, and privacy concerns. 
This paper proposes an alternative activity tracking and small-scale localization method based on inverse use of 3D digital compass sensors. In our approach, a number of magnetometer sensors are placed within the monitored environment in order to detect magnetic interference introduced by a simple permanent magnet carried by the study subject (human or animal). Digital compass sensors are low-cost, low-power with minimal data processing requirements. Permanent magnets, in turn, do not require batteries and can be safely worn for prolonged periods of time (for example, as a bracelet) [1]. Magnetic tracking is less intrusive than surveillance cameras and does not require line of sight.

The rest of the paper is organized as follows. The next section provides a brief overview of related work on micro-localization and activity tracking methods and their limitations. Then we introduce the concept of magnetic tracking and discuss its benefits, limitations and open challenges. The paper concludes with the early results of proof-of-the-concept experiments.

\section{Related work}

With the advance of technology, activity tracking studies employed increasingly sophisticated methods, ranging from direct observation and surveillance cameras, to smartphones and wearables with motion sensors (accelerometers, gyroscopes) and more recently device-free localization $[2,3]$.

Video-based monitoring provides rich and accurate information for analysis. However, these benefits come at the expense of user's privacy. Moreover, the observation area of the video-based tracking is limited by the view field of the cameras and eventual obstacles in the environment (such as furniture). Furthermore, automated processing of the video stream requires considerable computational resources (or communication bandwidth), making battery-powered wireless solutions unfeasible.

Smartphones are suitable for large-scale monitoring, but in home and office settings these mobile devices are often left stationary [4], which limits their utility for small-scale indoor activity tracking. Moreover, the variety of phone carrying options (in belt holster, or front, side, or back pocket) further complicates processing of motion activity data.

Wearable sensors, such as fitness trackers and smart watches, solve the last issue and allow continuous tracking outdoors, indoors and even while the user sleeps. Unfortunately, the need for frequent (hours to days) charging limits the utility of wearable trackers: the user may forget to charge the device or to wear the device after charging. Moreover, the procedure may not be feasible for technology-reluctant elderly people [5].

A promising solution to the issues described above is device-free localization (DFL) $[2,3]$, where the location of the user is inferred from the effect of human body on radio wave propagation. DFL systems do not require any wearables (and hence batteries), but can be affected by radio interference and environmental changes. Unlike video-based solutions (which also fit into the device-free definition) radio-based DFL systems are more privacy-friendly, since they can only provide location and activity data and do not use visua information at any stage. Unfortunately, the resolution of the existing DFL systems is limited by the number of deployed sensors [2] and physical laws of wave propagation 


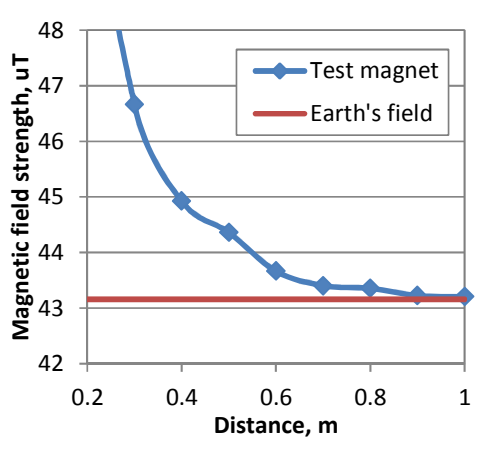

Figure 1. Magnetic field strength of a small neodymium magnet $(10 \times 8 \mathrm{~mm}$ cylinder) as measured by the digital compass of a Google Nexus 7 tablet.
Some indoor positioning systems (e.g., [6]) employ modulated magnetic fields; however, mobile clients of these systems are active, battery-powered devices.

Tracking of permanent magnets by digital magnetometers has previously been demonstrated as an interaction method for mobile devices. Ketabdar et al [7] proposed a mobile system controlled by the gestures of a user wearing a magnetic ring. Afshari et al [8] demonstrated a magnetic joystick for mobile gaming, showcasing the centimeter-scale resolution of the magnetic sensing. Present paper explores the idea of magnetic tracking in the broader scope of ambient assisted living and indoor localization.

\section{Passive magnetic sensing}

Magnetic tracking combines the accuracy of videobased methods with the privacy and battery-free benefits of DFL systems. In the proposed approach, the subject carries a permanent magnet (for example, as a bracelet, collar or integrated into the clothes). The environment is equipped with several stationary sensing nodes with magnetometers, placed in key areas of interest. The magnetic field strength measured by each sensor is defined by the Earth's global magnetic field and eventual local anomalies (for example, metal building frame) and is normally stable. Subject's movement near the sensor node changes the distribution of the magnetic field and can thus be detected. Since the magnetic field strength quickly decreases with the distance (see Figure 1), the node can detect magnet's presence only in the close vicinity (few decimeters for normal magnets, up to a meter for small neodymium magnets)
The wearable component of the magnetic tracking system is a completely passive permanent magnet which is the main benefit of the proposed approach. Magnets are cheap, do not require batteries, can be easily embedded into jewelry or clothes. On the other hand, strong magnets may interfere with daily activities of the subject and affect navigation devices nearby. Also, user identification would require additional equipment (such as RFID). Finally, the user may remove the magnet and thus become invisible for the system (which, however, can be advantageous in certain use cases).

Stationary magnetometer sensors, in turn, have low power consumption and simple outputs which can easily be processed by a battery-powered microcontroller. Unlike acceleration data that need to be sampled at a high rate, magnetic transitions are relatively slow and can thus be recognized at a considerably lower sampling rate, which reduces the energy consumption even further. In contrast to surveillance cameras which involve high data rates and require complex processing, magnetic sensors have two advantages. Firstly, they do not require line of sight and can thus be hidden under any non-conductive surface (such as inside wooden or plastic furniture, doorframes, tables). Secondly, they do not invade user's privacy and can be safely used in sensitive areas such as bathrooms. In addition, they are indifferent to humidity and varying lighting conditions which have a serious impact on video-based methods. In comparison to RFID, magnetic sensors are considerably more compact than RFID reader antennas and consume less power. 


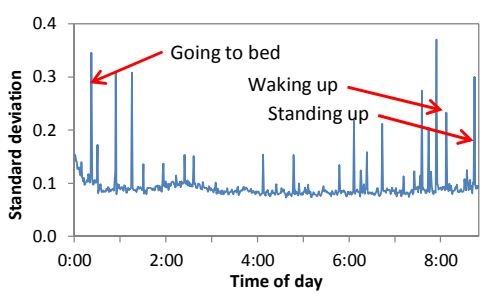

Figure 2. Sleep pattern monitoring (standard deviation of the magnetic field strength within 1-minute windows).

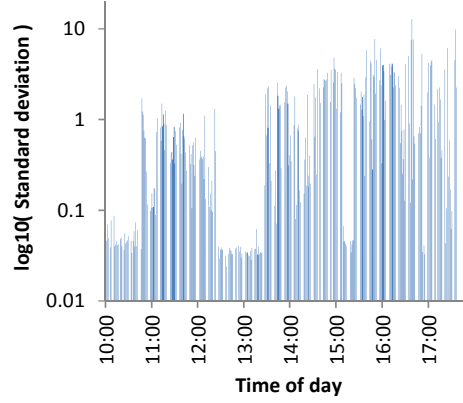

Figure 3. Office desk activity log (meeting until 10:40; lunch from 12:30 to 13:30; ten-minute coffee breaks at $15: 10$ and $16: 50$ )
However, magnetic tracking has a number of general limitations. Firstly, permanent magnets do not have unique signatures, so the magnetic tracking will need to be combined with some user recognition system when user identity is important. Secondly, while detection of simple movements and transitions is fairly simple, precise tracking of magnet location is more challenging. Finally, the monitored area of the magnetometer is limited by its sensitivity and magnet's strength. The latter, in turn, is limited by practical considerations, since the person wearing an overly strong magnet would attract all the magnetic objects on their way, including cutlery. From our tests, practical distance is limited to $0.5-1 \mathrm{~m}$ (see Figure 1), which is sufficient for localized monitoring of key areas such as doorframes, beds or medical cabinets.

\section{Proof-of-the-concept experiments}

The feasibility of the proposed approach has been evaluated in two simple experiments with a small neodymium magnet and a 3D compass of the Google Nexus 7 tablet (sampled at $5 \mathrm{~Hz}$ ). User activity was estimated as the standard deviation of magnetic field strength within non-overlapping 1-minute windows.

The first experiment focused on sleep tracking. A magnetic bracelet was worn on a wrist, while the tablet was placed under the bed (about $0.5 \mathrm{~m}$ from the magnet). Similarly to the actigraph devices used in sleep studies [9], digital compass was able to detect movements of the sleeping person (Figure 2).

In the second experiment, the tablet was placed on an office desk to monitor work activity. Logged data (Figure 3 ) show intervals when the subject was away from the desk.

\section{Acknowledgements}

This work was supported by the National Research Fund of Luxembourg (grant number C14/IS/8311593 INDOORS project).

\section{References}

[1] Schenck, J.F. Safety of strong, static magnetic fields. J Magn. Reson. Imaging 2000; 12:2-19.

[2] Wilson, J., Patwari, N. Radio tomographic imaging with wireless networks. IEEE Trans. Mob. Comput. 2010. 9(5), 621-632.

[3] Sigg, S., Scholz, M., Shi, S. et al. RF-sensing of activities from non-cooperative subjects in device-free recognition systems using ambient and local signals. IEEE Trans. Mob. Comput. 2014, 13(4), 907-920.

[4] Dey, A.K., Wac, K., Ferreira, D., Tassini, K., Hong, J.H., Ramos, J. Getting closer: an empirical investigation of the proximity of user to their smart phones. Proc. UbiComp-2011.

[5] Mahoney, E.L. and Mahoney, D.F. Acceptance of wearable technology by people with Alzheimer's disease: issues and accommodations. Am J Alzheimers Dis Other Demen. 2010 Sep; 25(6):527-31.

[6] Pirkl, G., Lukowicz, P. Robust, low cost indoor positioning using magnetic resonant coupling. Proc. UbiComp-2012.

[7] Ketabdar, H., Yuksel, K.A., Roshandel, M. MagiTact: interaction with mobile devices based on compass (magnetic) sensor. Proc. IUI-2010.

[8] Afshari, S., Popleteev, A., McCall, R., Engel, T. Magnetic interaction with devices: A pilot study on mobile gaming. Proc. NordiCHI-2014.

[9] Ancoli-Israel, S., Cole, R., Alessi, C. et al. The role of actigraphy in the study of sleep and circadian rhythms. American Academy of Sleep Medicine Review Paper. SLEEP 2003; 26(3):342-92. 